\title{
Strategies for a Safe Integration of Water-Bearing MEP Systems in Prefabricated CLT Room Modules
}

\author{
Michael Monsberger ${ }^{*}$, Gerhard Schickhofer ${ }^{2}$, Anna-Katharina Magg ${ }^{3}$, Konstantin Ganster ${ }^{4}$, \\ Markus Neumayer ${ }^{5}$, Thomas Bretterklieber ${ }^{5}$, and Christian Kaufmann ${ }^{6}$ \\ ${ }^{1}$ Professor, Institute of Construction Management and Economics, \\ ${ }^{2}$ Professor, Institute of Timber Engineering and Wood Technology, \\ ${ }^{3}$ Junior Researcher, Institute of Construction Management and Economics, \\ ${ }^{4}$ PhD candidate, Institute of Timber Engineering and Wood Technology, \\ ${ }^{5}$ Senior Researcher, Institute of Electrical Measurement and Measurement Signal Processing, \\ Graz University of Technology, Austria \\ ${ }^{6}$ Managing Director, Kaufmann Bausysteme GmbH, Austria \\ "Corresponding author's e-mail: michael.monsberger@tugraz.at
}

\begin{abstract}
The market for modular buildings based on prefabricated room modules made of cross-laminated timber (CLT) is rapidly growing in Europe. Short construction times combined with high construction quality due to prefabrication are highly attractive features of this construction method. While structural design aspects have been steadily improved, only little attention has been paid to mechanical, electrical and plumbing (MEP) systems so far. However, the integration of waterbearing pipes and surface seals must be done with great care, since a permanent exposure of the timber construction to moisture caused by leaks may result in biological degradation of the wooden structure. The risk of moisture damage can be reduced by decreasing the length of horizontal piping by means of intelligent pipe routing. In addition, sensor technology can be used to detect water leaks in critical areas where other measures fail to provide sufficient protection. Both aspects are addressed in this paper. First, the results of an analysis of nine completed construction projects based on CLT modules including hotels, student residences, nursing homes, and health care centers are presented. The projects were used to identify the current state of practice regarding the design of CLT room modules with respect to the integration of MEP installations. In a next step, general strategies for a safe integration of MEP installations in timber buildings are outlined. A bathroom layout with optimized pipe routing based on three individual shafts is chosen to show the application of these strategies. The approach enables a considerable reduction of horizontal pipe length while maintaining the functional and architectural requirements of the room module. Finally, a new planar sensor for detecting water leaks is presented. The results were developed in the research project SensGT and will lead to standardized solutions for a safe integration of MEP installations in CLT room modules.
\end{abstract}

\section{KEYWORDS}

Room modules; Cross-laminated timber; MEP integration; Moisture sensor 


\section{INTRODUCTION}

Modular buildings based on cross-laminated timber (CLT) room modules are gaining more and more attention (Ferdous et al., 2019). This construction method is particularly suited for buildings with a high number of repetitive rooms, such as hotels, student residences, nursing homes, etc. Recently, also multi-family housing projects have been successfully launched. Figure 1a shows a production facility for prefabricating room modules made of CLT. The prefabricated modules are fully equipped with furniture and bath facilities (Figure 1b) before they are brought to the construction site by trucks and assembled (Figure 1c). The capacity of such prefabrication facilities in Austria ranges up to 2000 modules per year per plant. While research efforts have been focused on aspects such as structural design, joining techniques, and building physics (e.g., Brandner et al., 2016, Merz, 2018, Buchegger et al., 2018), almost no attention has so far been paid to the integration of mechanical, electrical, and plumbing (MEP) systems. For this reason, current MEP installation practices in room modules do not take into account possibilities offered by modularity and prefabrication; neither do they provide precautionary measures to protect the wooden structures from potential water leaks. This paper mainly addresses the second issue. Undetected exposure of timber structures to water over longer periods of time can lead to biological degradation of the wooden structure and cause substantial damage (Schickhofer and Schmid, 2014). Leaking water pipes or damaged surface seals (e.g., in bathrooms) are potential sources of such water exposure. The goal of this paper is to highlight the relevance of a safe integration of water-bearing MEP installations in timber buildings and to discuss approaches of how such an integration can be achieved using modular buildings as an example. The paper is divided into three main sections. The first section provides an overview of the current state of practice in Austria and Germany. Nine completed modular building projects are analyzed for this purpose. The second section introduces general approaches for a safe integration of water-bearing MEP systems in timber buildings. In the third section, two specific approaches, the minimization of horizontal pipe length and the use of sensor technology to detect water leaks, are discussed in detail.
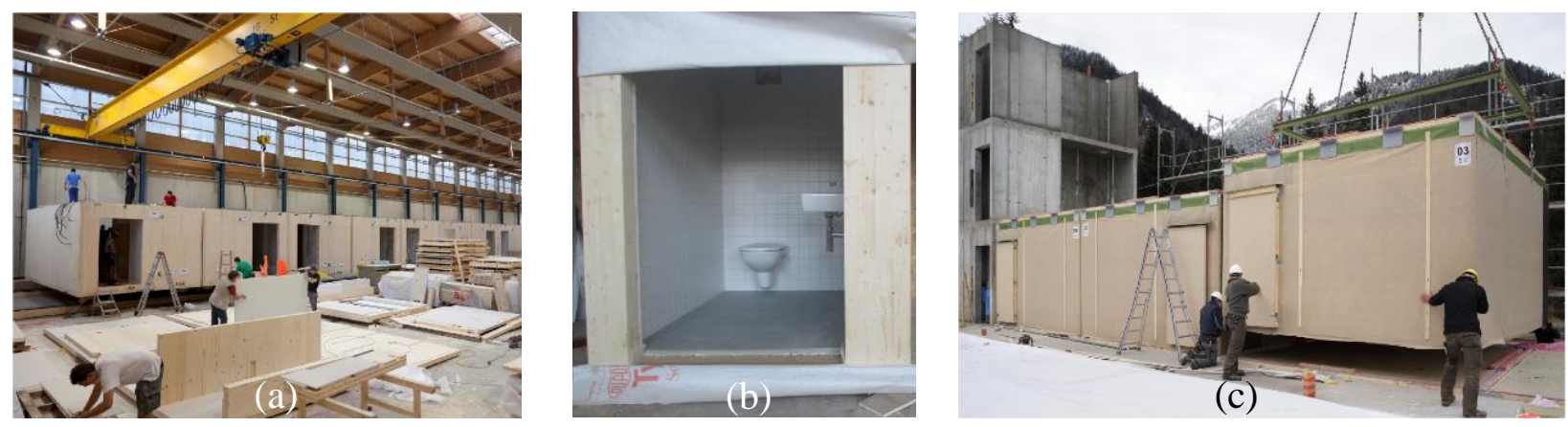

Figure 1. (a) Production facility of CLT room modules in Austria; (b) fully equipped bathroom inside a module; (c) module assembly at the construction site.

\section{ANALYSIS OF COMPLETED PROJECTS IN AUSTRIA AND GERMANY}

A comprehensive analysis was conducted in order to determine the current state of practice regarding the design of CLT room modules with respect to the integration of water-bearing MEP installations. Results obtained for plumbing are presented in the following. Nine projects in Austria and Germany were evaluated by means of the following criteria: room module layout, bathroom layout (placement of sanitary appliances), shaft positions, and pipe routing. Each project uses one 
or more standard room module types (representing the vast majority of modules used in a building) as well as special types (e.g., rooms for handicapped people). Twelve standard room module types were identified in the nine considered projects. Figure 2 shows the bathroom layouts of these modules. In projects 1 and 2, the bathroom layouts are of the same type.

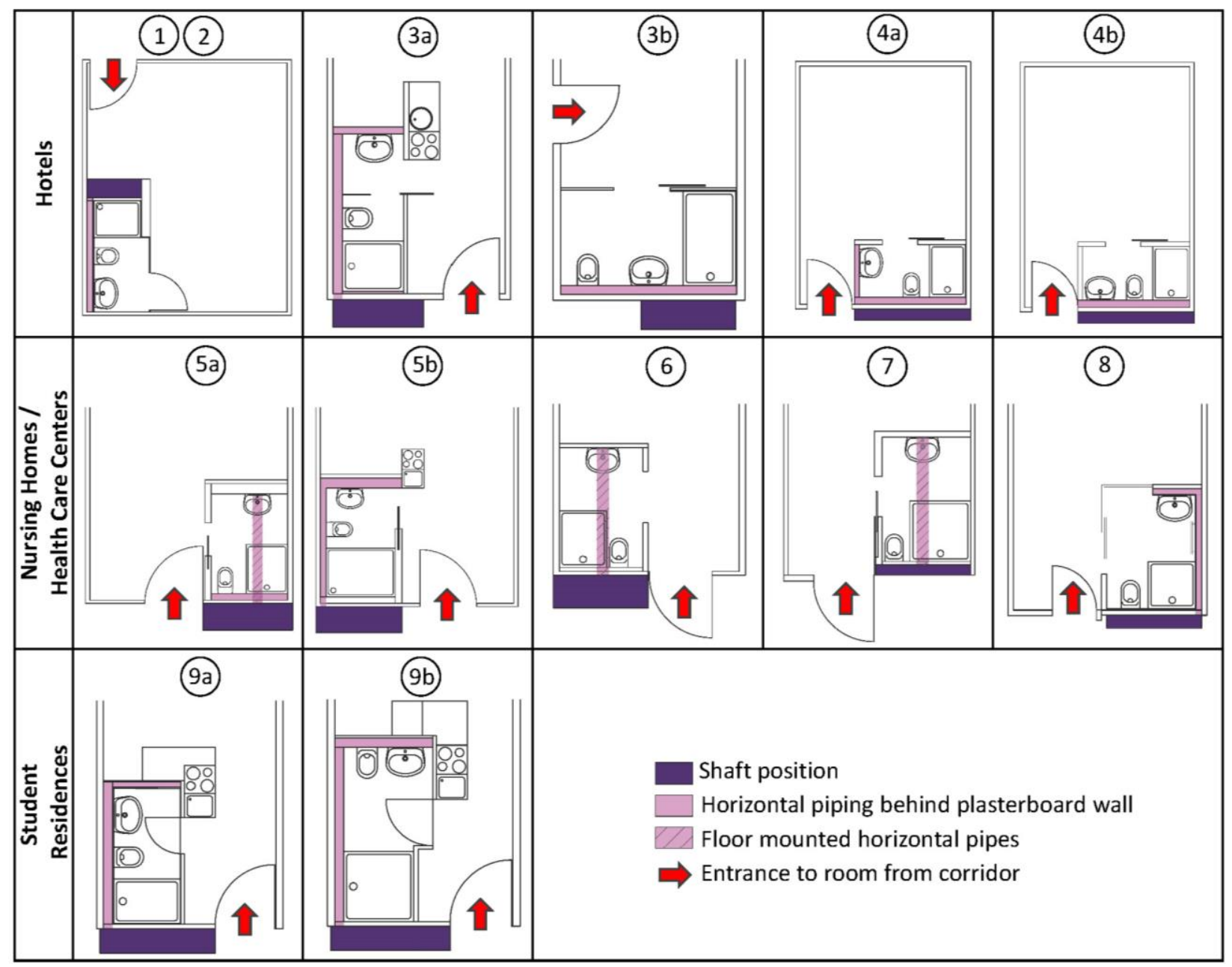

Figure 2. Bathroom layouts of the standard room modules of the analyzed projects.

The bathrooms of all room modules are equipped with the following appliances: a toilet, a shower, and a sink. Modules 5b, 9a, and $9 \mathrm{~b}$ also include a small kitchen. The bathroom is usually located next to the entrance door, either on the left or the right hand side. The piping largely depends on the shaft position and the location of the appliances. With exception of modules 1 and 2, the shaft position is commonly at the wall between the bathroom and the corridor (usually outside the module, i.e., on the corridor side). This arrangement offers the advantage that pipe connection and completion of the shaft can be carried out at the construction site without entering the furnished room modules. Most modules use plasterboard walls in combination with in-wall carriers and mounting frames for a wall-hung installation of the appliances. The appliances are connected to each other and to the shaft by means of horizontal piping. As illustrated in Figure 2, horizontal pipes were either installed behind plasterboard walls or floor mounted. The horizontal pipe length depends on the positions of the appliances. Short pipe length and few fittings minimize the risk of water damage. In this regard, the arrangement of module $4 \mathrm{~b}$ is advantageous, as all appliances are 
directly located at the shaft. The same approach was used for the installation of the shower and toilet in modules 5a, 6, 7, and 8. However, horizontal piping across the entire bathroom is required to connect the sink in these cases. In modules 1, 2, and 9a, the three appliances are installed in a row. Figure 3 exemplarily shows the practical implementation of module 9a: The pipe routing is well structured and all pipes are installed behind a plasterboard wall (no floor-mounted pipes, which is favorable). The concentrated installation reduces the area where potential leaks may occur and facilitates the implementation of additional measures to protect the timber structure against water leaks. However, such measures are not yet implemented. It is apparent from Figure 3 that all pipes are individually mounted on the backside wall. A prefabricated element is only used for the installation of the toilet.

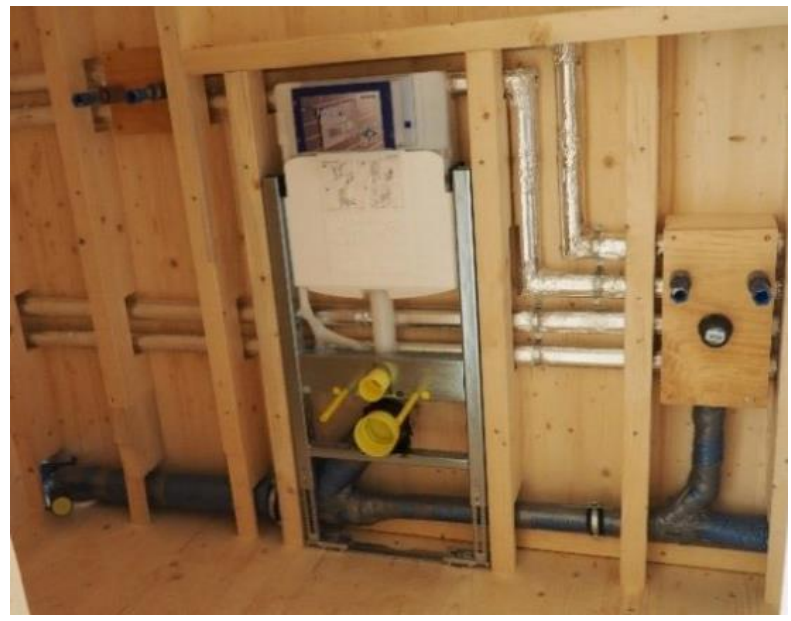

Figure 3. State-of-the-art pipe routing behind a plasterboard wall (room module 9a in Figure 2).

\section{STRATEGIES FOR A SAFE INTEGRATION OF MEP INSTALLATIONS}

The above analysis as well as experience show that MEP installations in Austria and Germany are made according to current state-of-the-art rules. However, these rules evolved from practices related to traditional construction methods (concrete, brick, steel). Tailored solutions taking into account requirements of but also possibilities offered by (modular) timber construction have not yet been established. Hausladen and Schickhofer were among the first authors to address this topic. Hausladen et al. (2008) investigated and developed specific MEP solutions for modular timber buildings. They also formulated six general principles for the integration of MEP installations in modular timber buildings. Schickhofer and Schmid (2014) enhanced the principles and highlighted the importance of a safe integration of water-bearing MEP installations in timber buildings. They also suggested design solutions for such an integration. Based on these works, the following general approaches, which facilitate a safe integration of MEP in (modular) timber buildings, can be derived:

Concentrated pipe routing with reserve space: Pipe routing should be concentrated in welldefined spaces. This leads to the reduction of areas where water leaks may occur and facilitates the implementation of precautionary measures to protect timber structures. Reserve spaces facilitate future extensions and simplify inspection and maintenance works. 
Reduction of pipe length and pipe joints: An optimized arrangement of shafts and sanitary appliances enables a reduction of the required pipe length, which also leads to a reduction of joints. This minimizes the number of spots where water leakage may occur and thus reduces areas to be protected and controlled.

Adequate routing of water-bearing pipes: Water bearing-pipes should be installed in such a way that water from potential leaks can easily be spotted and that it does not migrate into the timber structure immediately. Preferable options include visible on-wall installations, installations in suspended ceilings or in installations behind skirting boards. If such options are not possible, additional protective measures should be considered.

Integration of protective measures: Pipes that are routed in inaccessible areas (e.g., behind or inside walls) should be protected by additional measures. Appropriate protective measures may be troughs, protection plates, additional seals, tube-in-tube solutions, etc.

Accessibility, inspection, and monitoring of installation lines: Accessibility to installation lines (e.g., via inspection flaps or maintenance doors) enables frequent inspections to detect potential leaks and damage, which is an effective way to prevent water and moisture damage. Inaccessible areas (e.g., areas underneath shower trays) may be electronically monitored using moisture sensors. However, such electronic monitoring systems are not yet state-of-the-art.

The analysis presented above shows that some of these aspects are already taken into account (e.g., positioning of appliances close to shafts, concentration of pipe routing behind plasterboard walls). However, specific measures to protect timber structures are currently not implemented. A main barrier is that readily available and easy-to-use standard designs and solutions are still missing. Buildings made of room modules are a well suited use case for the development of such solutions as their practical implementation can be achieved by means of standardized and ideally also prefabricated MEP modules. In addition, the room layouts of these buildings are usually simple. This simplifies the practical application in a first step.

\section{CONCEPTS DEVELOPED IN THE RESEARCH PROJECT SensGT}

The research project SensGT focuses on the development of solutions for a safe integration of MEP installations in CLT room modules. The general principles introduced above form the basis of this work. The project has two specific goals. First, the design and experimental evaluation of optimized and standardized MEP installation elements for CLT room modules and second, the development and proof of concept of a novel low cost moisture sensor for detecting water leaks. Initial results of this work are presented below.

\section{Modular MEP elements for reducing horizontal piping}

Figure 4a shows a bathroom configuration typically found in modular buildings. The shower and the toilet are located at the shaft; the sink is located at the wall opposite the shaft (cf. modules 5a, 6, 7, 8 in Figure 2). In the example shown in Figure 4a (cf. module 8 in Figure 2), the horizontal pipes for connecting the sink are installed behind/within plasterboard walls. The shaft is located at the other side of the module (i.e., on the corridor). The resulting overall horizontal pipe length (hot and cold water supply and drain) of this particular example is around $14.7 \mathrm{~m}$. Figure $4 \mathrm{~b}$ shows an 
alternative approach pursued in the SensGT project. Each appliance has an individual shaft which carries vertical water and drain lines. The appliances are directly connected to these vertical lines, resulting in a substantially reduced overall length of horizontal pipes of around $0.8 \mathrm{~m}$. Of course, the overall length of vertical piping increases. However, the vertical pipes are concentrated within the shafts, which simplifies the use of inspection flaps, the implementation of protective measures inside the shafts or the application of monitoring devices. Prefabricated modules will be used to create the three shafts. The shaft modules are installed during assembly of the room modules in the plant and connected at the construction side.

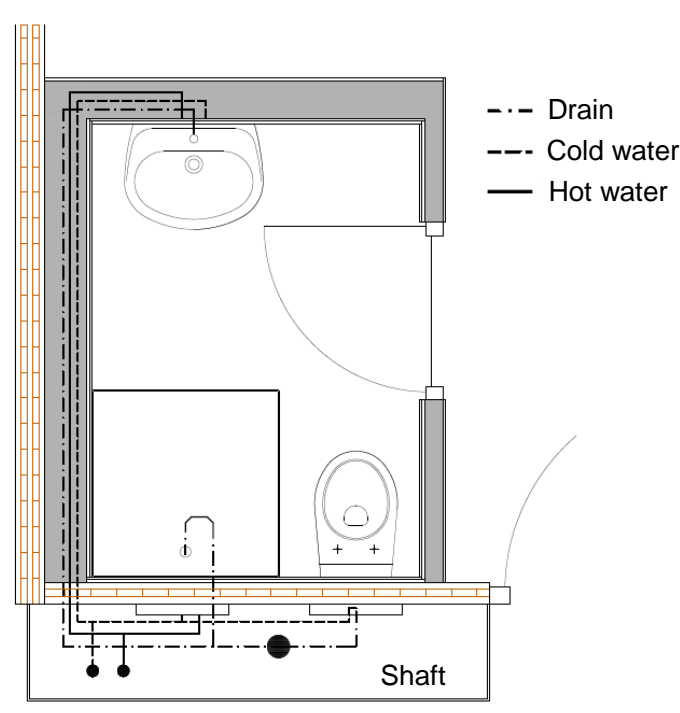

(a)

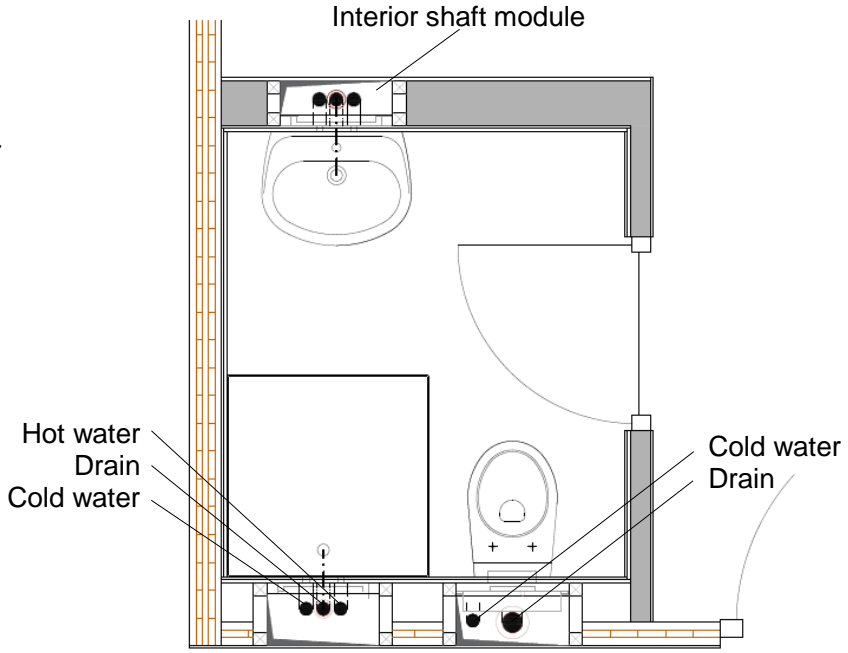

(b)

Figure 4. (a) Conventional pipe routing; (b) modular approach with reduced horizontal piping.

Within the SensGT project, the shaft modules are designed, built, and evaluated on lab-scale by means of complete bathroom mock-ups. Design criteria include fire protection and building physics requirements as well as protection of the timber structure against water leaks. A focus is also put on details such as connection of interior shafts at the construction site. This concept is one specific approach that is investigated among others in the SensGT project.

\section{Distributed passive detector for moisture sensing}

For areas which are difficult to protect by means of design measures (e.g., areas behind shower walls or under shower trays), the use of electronic monitoring equipment is a viable option for the future. For this reason, a novel system (patent pending) for water and moisture sensing in structures is developed in the SensGT project. Several technical challenges have to be addressed including large sensing areas, harsh environment, and long lifetime. In addition, the solution must be low cost and simple to install. The effort for wiring the sensor has to be considered in this context. These aspects limit the applicability of classical sensing techniques, as these sensors only provide local measurements.

\section{Sensor element: Sensing and signal transmission principle}

To address these design criteria, the use of a capacitive sensing element and a wireless, passive transmission technique is proposed. Figure 5a depicts a sketch of a single sensor element (SE). 


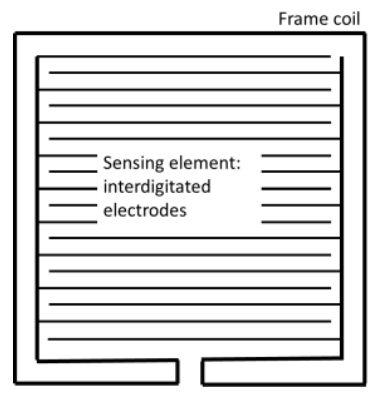

(a)

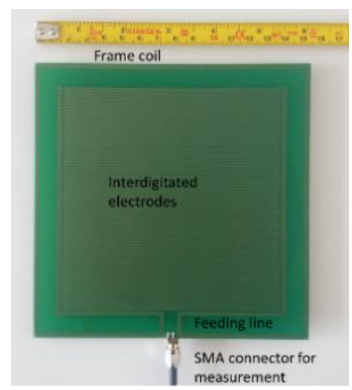

(b)

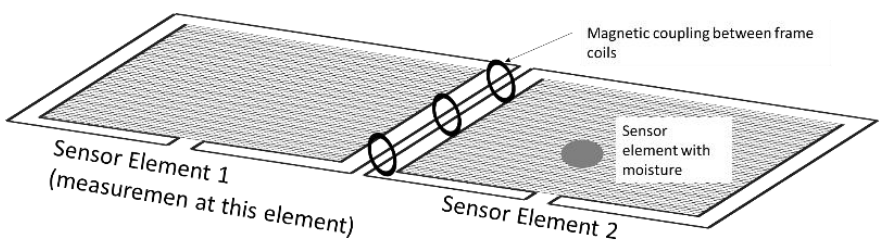

(c)

Figure 5. (a) Sensor element; (b) photograph of a sensor element; (c) coupling of sensing elements.

The capacitive sensor is realized by means of interdigitated electrodes (finger-type electrodes) encompassed by a frame coil. Moisture or water droplets on the sensor will influence the coupling capacitance $C$ between the electrodes. The frame coil has the inductance $L$. Both elements form a resonance circuitry with the resonance frequency $f_{-}$res $\approx \sqrt{ }(L / C)$ (Ida, 2015). Figure $5 \mathrm{~b}$ shows a photograph of a single SE. It is currently realized by means of a PCB board. The sensor readout, i.e., the measurement of $f_{-} r e s$ to determine the presence of moisture, can be performed by different techniques (e.g., impedance measurements). A single SE already covers a larger area than conventional sensor solutions. To further enlarge the sensing area, individual SEs are placed next to each other. Figure 5c illustrates the principle. SE1 is used for readout by connecting it to a readout device. SE2 is placed next to SE1. The magnetic coupling between the frame coils of SE1 and SE2 leads to a coupling of the individual resonance circuits. Moisture on SE2 can therefore be measured via SE1. This concept can be used to extend and shape the sensing area.

\section{Lab Demonstration}

Figure 6 shows an experiment with six SEs placed next to each other. The chain spans a distance of about $1 \mathrm{~m}$. SE1 is used as readout element and water is applied on SE6 at the opposite end of the chain. A network analyzer was used for the readout, i.e., measuring the impedance at SE1. From the readout, the presence of water can clearly be detected as shown in Figure 6 . The proofof-concept for the application of this sensor concept in timber buildings will be made by integrating and testing it in the aforementioned bathroom mock-ups.

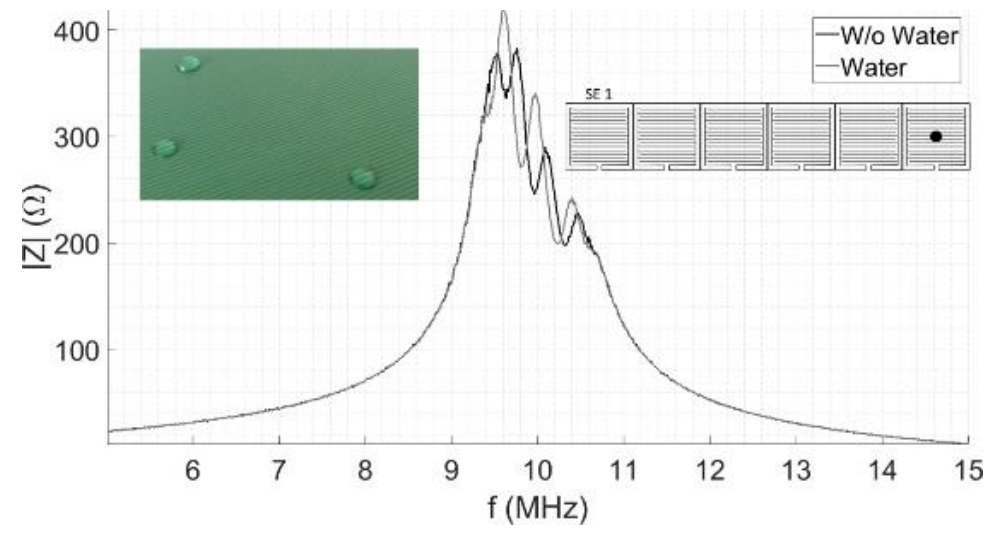

Figure 6. Experiment with a sensor chain consisting of six elements. The photograph shows the water placed on the last sensor element. 
MOC SUMMIT / MAY 2019

\section{CONCLUSION}

Undetected water leaks caused by MEP installations can lead to substantial damage in timber structures. Experience as well as an analysis of projects in Austria and Germany show that stateof-the-art MEP installations in timber buildings neither include measures to protect wooden structures nor utilize design and prefabrication options offered by modular building construction. A safe integration of MEP installations in timber buildings is achieved by consistently applying the principles and strategies described in this paper and the related literature. A barrier that currently limits their practical application is the lack of easy-to-use standard designs and solutions that can be readily used by practitioners. Modular buildings made of CLT room modules represent a well suited pilot use case for developing such solutions as this construction method features repetitive and simple room layouts. Two specific approaches developed in the research project SensGT were presented and discussed in this paper. The first approach uses prefabricated shaft modules in which the piping required to connect the sanitary appliances is concentrated. This way, horizontal pipe routing along timber walls or on the floor is avoided. The second approach uses sensors to detect water leaks. A novel passive sensor concept capable to provide the required functionality was introduced. Even though such works represent a first step towards a safer integration of MEP installations in timber buildings and standardized solutions, more efforts are required by science and industry to make the presented principles good engineering practice.

\section{ACKNOWLEDGEMENTS}

The presented work was done within the framework of the Bride 1 project SensGT (Grant No. 864724) funded by the Austrian Research Promotion Agency (FFG).

\section{REFERENCES}

Brandner, R., Flatscher, G., Ringhofer, A., Schickhofer, G., and Thiel, A. (2016). "Cross laminated timber (CLT): overview and development." European Journal of Wood and Wood Products, 74(3), 331-351.

Buchegger, B., Ferk, H., and Schanz, M. (2018). "Modal analysis and flanking sound insulation in connected panels of cross-laminated-timber at low frequencies." In Euronoise 2018 Conference Proceedings, May 2018, 1541-1548.

Ferdous, W., Bai, Y., Ngo, T.D., Manalo A., and Mendis P. (2019). "New advancements, challenges and opportunities of multi-storey modular buildings - A state-of-the-art review." Engineering Structures, 183, 883-893.

Hausladen, G., Huber, C., and Hilger, M. (2008). Holzbau der Zukunft Teilprojekt 12. Modulare, vorgefertigte Installationen in mehrgeschossigen Holzbauwerken. Fraunhofer IRB Verlag, Stuttgart, Germany.

Ida, N. (2015). Engineering Electromagnetics. $3^{\text {rd }}$ Ed., Springer International Publishing

Merz, K. (2018). "Statisch-konstruktive Überlegungen bei der Verwendung industriell vorgefertigter Raumzellen-Bausysteme." In Vorfertigen und Modularisieren im Holzbau, 3. Klagenfurter Holzbau-Fachtagung. Verlag der Technischen Universität Graz, Austria.

Schickhofer, G., and Schmid, G. (2014). "Gebäudetechnik für Geschoßbauten in HolzMassivbauweise." In Versorgen und Umhüllen im Holzbau. Schnittstellen des Holzbaus zur Gebäude- und Fassadentechnik, 1. Klagenfurter Holzbau-Fachtagung. Verlag der Technischen Universität Graz, Austria. 